\title{
Spectra of Wideband Dipole Radiation Induced by the Photomagnetoelectric Response in Narrow Gap Semiconductors
}

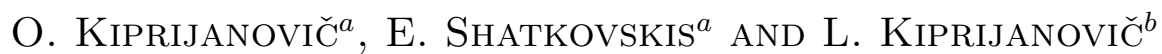 \\ ${ }^{a}$ Semiconductor Physics Institute \\ Goštauto 11, LT-01108 Vilnius, Lithuania \\ ${ }^{b}$ Faculty of Mathematics, Vilnius University \\ Naugarduko 24, LT-03225, Vilnius, Lithuania
}

(Received February 28, 2008; in final form April 28, 2008)

\begin{abstract}
The frequency spectra of sign inverted photomagnetoelectric responses in narrow gap semiconductors $\mathrm{InSb}$, InAs, and $\mathrm{Cd}_{0.2} \mathrm{Hg}_{0.8} \mathrm{Te}$, excited by nanosecond laser light pulses, were used for calculation of induced electromagnetic radiation frequency spectra in dipole approximation. The parallelepiped shape sample was considered as capacitor-like point dipole. The known Fourier transform property was used in calculations. Features of double sign inverted signals formation and its spectra are considered and compared with experimental results. The radiation of pulses, having spectra in terahertz range when excited by picosecond laser pulse from capacitor-like radiators that demonstrate double sign inverted photoresponses, is expected.
\end{abstract}

PACS numbers: 42.65.Re, 71.55.Gs, 75.50.Pp

\section{Introduction}

Recently considerable research efforts has been committed to generation and investigation of wideband and ultra-wideband short electromagnetic pulses including $\mathrm{THz}$ range using optic pulse excitation [1-3]. Radiation of electromagnetic pulses very often occurs in conditions that are similar to dipole radiation. Fast changes of the electric or magnetic dipole moments induce pulsed electromagnetic radiation, power of which is proportional to square of second time derivative of a dipole moment [4]. Wideband and ultra-wideband electromagnetic pulses draws attention because of their wide frequency spectra and may be used in various fields for many practical applications. 
Fast sign inverted signals are of specific interest because they have large values of second time derivative. Spectra of double sign inversion response that occurred due to photomagnetoelectric (PME) effect in narrow-gap semiconductors InSb, InAs, and $\mathrm{Cd}_{0.2} \mathrm{Hg}_{0.8} \mathrm{Te}$ were investigated in Refs. [5, 6]. It was revealed that the Fourier images of the double sign inverted signals consist of low and high frequency parts. The main power is concentrated in the low frequency part, which consists of two low frequency bands: the first 0-25 $\mathrm{MHz}$ band (with non-zero component at zero frequency) and the second 25-200 MHz band (with maxima at 60-80 MHz) [6]. Amplitude values of subsequent high frequency bands (high frequency part) constitute $0.6-5 \%$ of the maximal amplitudes of low frequency parts. High frequency part stretched up to $1 \mathrm{GHz}$ and showed slow downtrend with frequency growth. The response appears on open electrodes of the parallelepiped shape sample in transverse magnetic field under strong pulsed laser excitation. The sample is considered as a capacitor-like point dipole, in which the displacement current replaces the electron current of a wire dipole radiator. Such dipole radiation is rarely discussed in antenna theory [7].

This work is aimed to study wideband spectra of radiation induced in the semiconductors by PME responses. Dipole radiation spectra is calculated using the Fourier transform property, comprising of replacement of the $n$-th order derivative operator in the time domain by multiplication by $(\mathrm{i} \Omega)^{n}$ in the frequency domain. Practical application of the obtained results is discussed.

\section{Sign inversion of emf due to multiple PME effect}

Illumination of narrow gap semiconductor by laser light, quantum energy of which corresponds to spectral region of fundamental absorption, results in appearing of a high density electron-hole pair (EHP) in surface layer. EHP density gradient causes diffuse current, with current density $j_{\mathrm{d}}$ along the direction of incident light (Fig. 1). In magnetic field, applied transversally to the light direction,

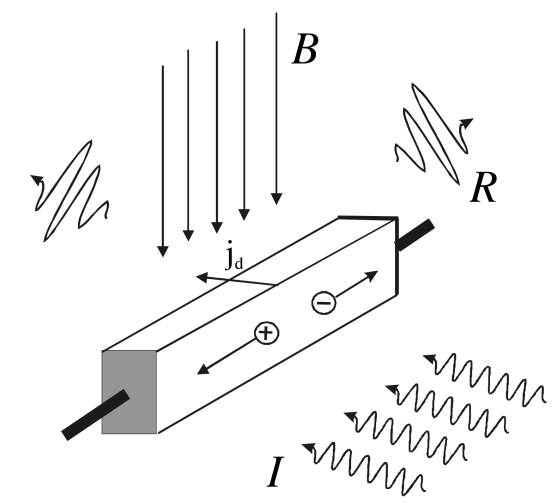

Fig. 1. Schematic diagram of the sample in crossed magnetic field $B$ and light beam $I$ for observation of radiation $R$ induced by PME response. 


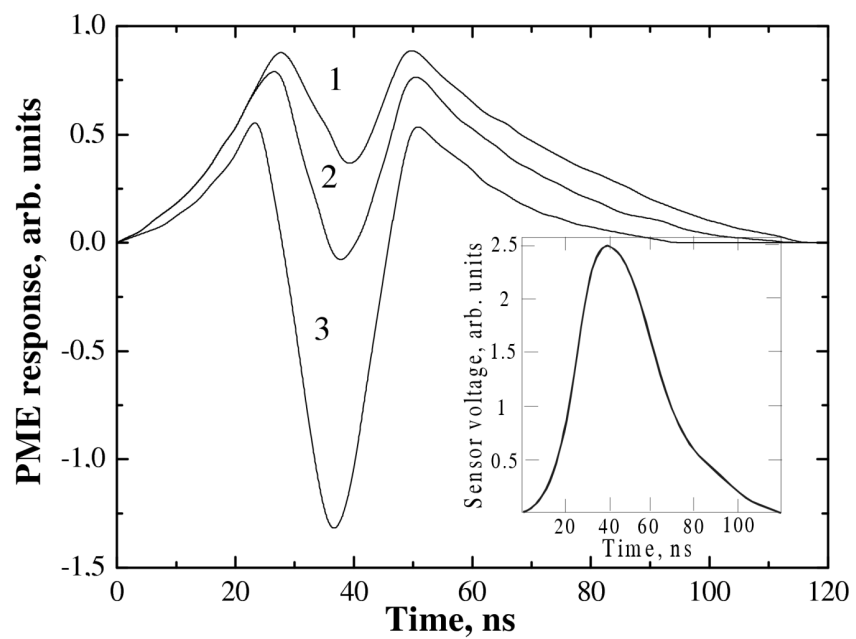

Fig. 2. The shape of the optically induced PME signal in $\mathrm{Cd}_{0.2} \mathrm{Hg}_{0.8} \mathrm{Te}$ for various light pulse intensities. $1-I<I_{\mathrm{c}}, 2-I=I_{\mathrm{c}}, 3-I>I_{\mathrm{c}}$. Optical pulse envelope is shown in the inset.

diffusing carriers are deflected to contact areas, creating electric field. This is the diffuse Kikoin-Noskov effect. Another effect, which appears under strong excitation, is EHP temperature gradient, directed along the diffuse current. The EHP temperature gradient causes appearance of electric field according to electronic analogue of the thermomagnetic Nernst-Ettingshauzen effect. Simultaneous occurrence of those two effects results in complex PME effect, creating sign inversion of electromagnetic force (emf) at the contact areas [8]. Figure 2 demonstrates $\mathrm{Cd}_{0.2} \mathrm{Hg}_{0.8} \mathrm{Te} \mathrm{PME}$ response time dependences with various laser pulse intensities. Optic pulse envelope (neodymium laser $h \nu=1.17 \mathrm{eV}$ ) is demonstrated in Fig. 2 inset.

In the narrow gap semiconductors the EHP electron component temperature excess $\Delta T_{\mathrm{e}}(I)$ at the surface can be well approximated by the formula

$$
\Delta T_{\mathrm{e}}(I)=A_{\mathrm{e}}\left(I / 10^{24}\right)^{\xi_{\mathrm{e}}},
$$

whereas EHP hole component temperature changes are insignificant up to light flux of $I \approx 10^{25}$ photon/ $\left(\mathrm{cm}^{2} \mathrm{~s}\right)$. Values of parameters $A_{\mathrm{e}}$ and $\xi_{\mathrm{e}}$ for the semiconductors [9] are presented in Table.

\section{TABLE}

The values of $A_{\mathrm{e}}$ and $\xi_{\mathrm{e}}$.

\begin{tabular}{c|c|c|c}
\hline \hline Parameters & InAs & InSb & $\mathrm{Cd}_{0.2} \mathrm{Hg}_{0.8} \mathrm{Te}$ \\
\hline$A_{\mathrm{e}}$ & 80 & 460 & 250 \\
$\xi_{\mathrm{e}}$ & 0.62 & 0.69 & 0.6
\end{tabular}


The kinetics of multiple PME effects is determined mainly by $\xi_{\mathrm{e}}$, as is shown in formula (1). The sign inversion of the response is observable at $I_{\mathrm{c}} \approx$ $(1-5) \times 10^{24}$ photons $/\left(\mathrm{cm}^{2} \mathrm{~s}\right)$. Value of $I_{\mathrm{c}}$ slightly increases with magnetic field enhancement. The most practically efficient case of full double sign inversion of PME emf when $I \gg I_{\mathrm{c}}$ (curve 3 in Fig. 2) has been analyzed in this work. The fastest signal rise time was observed in InSb crystals and the slowest one in $\mathrm{Cd}_{0.2} \mathrm{Hg}_{0.8} \mathrm{Te}$ ones. PME signal spectral components for the semiconductors were calculated using discrete Fourier transform (DFT) procedure and were presented in $[5,6]$.

\section{Results and discussion}

In the capacitor-like dipole displacement currents replace the electron current in a wire dipole radiator. The dipole is being shown as sample of length $l$ with charged contacts whose area is $S(\sqrt{S} \ll l)$. The dipole radiation is determined by the second derivative of dipole moment in the case when $l \ll \lambda$, where $\lambda$ is wavelength of radiation. The second derivative of the dipole moment is [7]:

$$
\frac{\mathrm{d}^{2}(q l)}{\mathrm{d} t^{2}}=\frac{l \mathrm{~d}^{2}(C \varphi)}{\mathrm{d} t^{2}}=l C\left(\frac{\mathrm{d}^{2} \varphi}{\mathrm{d} t^{2}}\right),
$$

where $C$ is the sample capacitance and $\varphi$ is potential difference which appears at the contact areas due to PME response. In the case when $\sqrt{S} / l \ll 1$ we use approximate formula from Ref. [10] for calculation of capacitance $C$ :

$$
C \approx \frac{C_{0}}{2}\left[1 /\left(1-\frac{C_{0}}{4 \pi \varepsilon_{0} \varepsilon l}\right)\right],
$$

where $\varepsilon_{0}$ is electric constant $8.856 \times 10^{-12} \mathrm{~F} / \mathrm{m}, \varepsilon$ is semiconductor permittivity ( $\varepsilon=12.7$ for $\mathrm{Ca}_{0.2} \mathrm{Hg}_{0.8} \mathrm{Te}, \varepsilon=14.5$ for InAs, and $\varepsilon=17.9$ for InSb), $C_{0}$ is capacitance of single square plate: $C_{0} \approx 8 \varepsilon_{0} \varepsilon a 0.566$ [10], $a=1 \mathrm{~mm}$ and $l=7 \mathrm{~mm}$.

Replacing the dipole moment with right side of expression (2) and converting the formula from Ref. [4] to the SI system, we obtain expression for spectral density of radiation intensity component $E_{n}$, which corresponds to PME signal spectral component $S_{n}[6]$ :

$$
E_{n}=\frac{\mu_{0}}{3 \pi c}(l C)^{2}(2 \pi n f)^{4}\left|S_{n}\right|^{2}
$$

where $n$ is DFT harmonic number, $f$ is the first harmonic frequency, $\mu_{0}$ is the magnetic constant, $c$ is the velocity of light.

The radiation frequency spectrum components $E_{n}$, obtained using formula (4), are presented in Fig. 3 for $\mathrm{Cd}_{0.2} \mathrm{Hg}_{0.8} \mathrm{Te}$ and InAs and in Fig. 4 for InSb. As can be seen from Figs. 3, 4 after application of formula (4) low frequency part of irradiated signal spectra does not contain first band, and main irradiated signal energy still concentrates in the remaining band. The values of central frequencies of these bands are increasing by a factor $\approx 1.66$ for the cases in Fig. 3 and $\approx 2.33$ for the case in Fig. 4 . In Fig. 3 one can observe a leveling of subsequent high frequency band amplitudes that now constitute $15-25 \%$ of the 
amplitude value of low frequency part, while PME response spectra show slow downtrend with increase in frequency. Also one can see in Fig. 4 a formation of single high frequency band with central frequency of $600 \mathrm{MHz}$ and constituting $26 \%$ of the low frequency part amplitude value. However, spectra of InAs and spectra of InSb have bands with high spectral density values at frequencies near $1000 \mathrm{MHz}$. This increase comes from the used theoretical model and the analyses of spectra of experimentally obtained signals do not show increase in high frequency component values $[2,3]$.

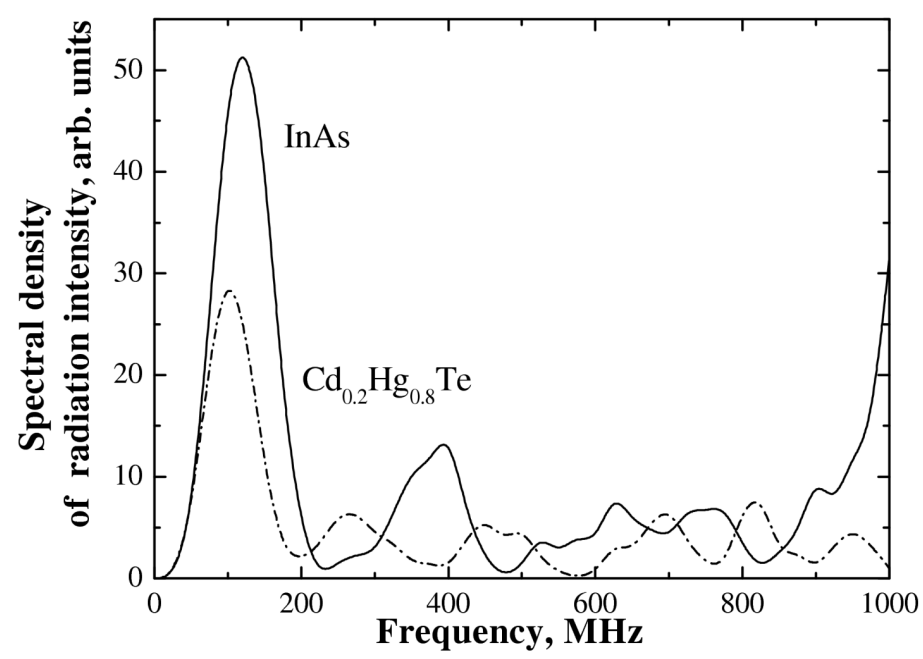

Fig. 3. Distribution of the spectral density of dipole radiation from $\mathrm{Cd}_{0.2} \mathrm{Hg}_{0.8} \mathrm{Te}$ and InAs samples.

Appearance of the increase can be explained as follows. Using combination of pair Gauss functions (smooth functions) $A_{1} \exp \left(-\beta_{1}^{2} t^{2}\right)-A_{2} \exp \left(-\beta_{2}^{2} t^{2}\right)$ for approximation of time sign inverted signal, one can obtain that this approximation after application of formula (4) to the Fourier transform of the signal well describe the spectrum of the irradiated signal in low frequency part, but high frequency components of irradiated signal spectrum disappear just after $300 \mathrm{MHz}$.

In contrast, using a broken line (having break derivative function) for time approximation of sign inverted signal, one can obtain that this approximation after application of formula (4) to the Fourier transform of the signal describe low frequency part of spectrum well too, but high frequency spectrum components show unreal amplitude growth just after the low frequencies. We suppose that spectrum of irradiated signal, obtained by formula (4) from the Fourier transform of real time response, corresponds to spectrum which we would obtain from experimentally registered signal in the $0-900 \mathrm{MHz}$ band.

To consider some advantage of the obtained results let us take the case of InSb, because irradiated double sign inverted signal experimentally observed 


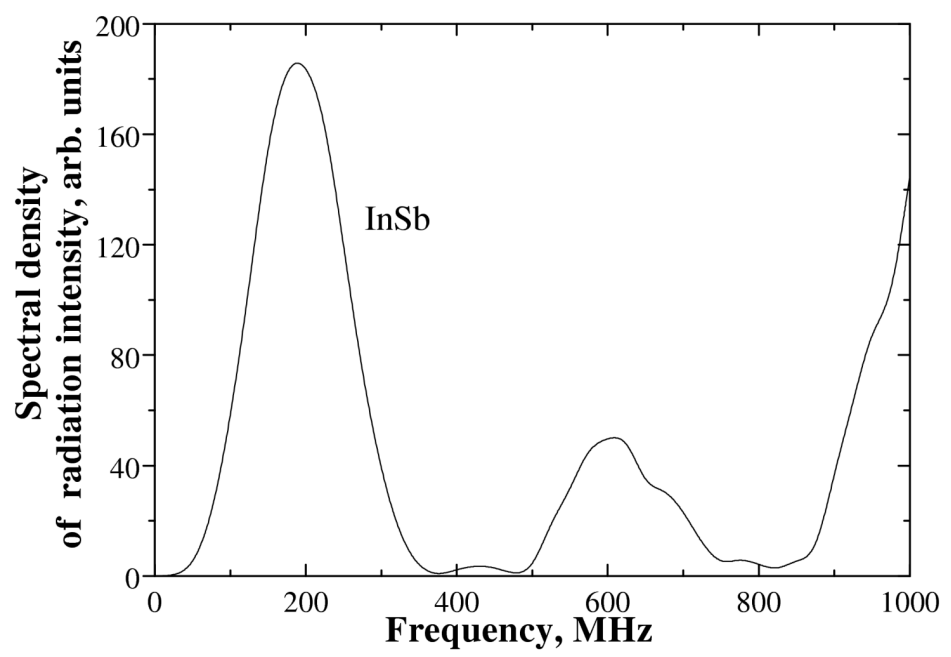

Fig. 4. Distribution of the spectral density of dipole radiation from InSb sample.

in Ref. [2] is homothetic to InSb PME response. This irradiated double sign inverted signal, which has $3.3 \mathrm{ps}$ between maxima and has $0.2-0.25 \mathrm{THz}$ maximal frequencies, is generated from film surface by $80 \mathrm{fs}$ optic pulse. As presented in Ref. [6], optical pulse, having envelope of $\approx 90$ ns duration at its base, generates in InSb double sign inverted response with 20 ns between the maxima (for comparison response of $\mathrm{Cd}_{0.2} \mathrm{Hg}_{0.8}$ Te in Fig. 2 has $48 \mathrm{~ns}$ ). Electron-hole pair kinetics remains unchangeable even when excited by femtosecond laser pulses $[1,3]$. Therefore, it is possible to generate similar double sign inverted response by $10-15$ ps laser pulse at capacitor-like sample with length smaller than $7 \mathrm{~mm}$. As a result, one should obtain spectra of radiated signal with about $0.55 \mathrm{THz}$ central frequency in low frequency part, because after application of formula (4) the central frequency in discussed case has increased by factor of 2.33 .

\section{Conclusion}

The parallelepiped shape sample of narrow gap semiconductor that demonstrates double sign PME response was considered as capacitor-like point dipole. Knowing the Fourier components of the PME signals and replacing the derivative operator of the second order in the time domain by multiplication by $(\mathrm{i} \Omega)^{2}$ in the frequency domain, the Fourier spectra components of the pulsed dipole radiation were found. The obtained results show that irradiated signals have wideband spectra enriched with high frequency components. The results also indicate a possibility of generation of wideband, including $\mathrm{THz}$ range, electromagnetic pulses using picosecond laser pulses and samples, which demonstrate double sign inverted photoresponses. 


\section{References}

[1] R. Adomavičius, A. Urbanowicz, G. Molis, A. Krotkus, Acta Phys. Pol. A 107, $132(2005)$.

[2] N. Kida, M. Tonouchi, Appl. Phys. Lett. 78, 4115 (2001).

[3] K.L. Yen, M. Hoffman, J. Hebling, K. Nelson, Appl. Phys. Lett. 90, 171121 (2007).

[4] L.D. Landau, E.M. Lifshitz, Theoretical Physics, Vol. 2, Field Theory, Nauka, Moscow 1973, p. 225.

[5] E. Shatkovskis, O. Kiprijanovič, A. Galickas, Acta Phys. Pol. A 107, 271 (2005).

[6] E. Shatkovskis, O. Kiprijanovič, Lithuan. J. Phys. 46, 229 (2006).

[7] T.C. Choy, Am. J. Phys. 72, 662 (2004).

[8] E. Shatkovskis, A. Tamaševičius, Phys. Status Solidi A 42, 111 (1977).

[9] E. Shatkovskis, Mater. Sci. Forum 297-298, 299 (1999).

[10] Y. Iossel, E. Kachanov, M. Strunskij, Calculation of Electric Capacitance, Energoizdat, Leningrad 1981, p. 117. 